05

\title{
Молекулярно-динамическое моделирование влияния кремния на упорядочение углерода в решетке мартенсита
}

\author{
(C) П.В. Чирков, А.А. Мирзоев, Д.А. Мирзаев
}

Южно-Уральский государственный университет, Челябинск, Россия E-mail: p.chirkow@gmail.com

Поступило в Редакцию 21 июля 2017 г.

Представлены результаты компьютерного моделирования влияния примесей кремния на степень тетрагональности и взаимодействия атомов углерода в ОЦК-решетке железа методом молекулярной динамики. Установлено влияние кремния на параметры решетки мартенсита, а также на основе теории упорядочения Зинера-Хачатуряна рассчитана зависимость от концентрации кремния параметра деформационного взаимодействия $\lambda_{0}$, определяющего критическую температуру ОЦК-ОЦТ-перехода. Обнаружено, что при увеличении содержания кремния до 10 at.\% происходит уменышение параметра $\lambda_{0}$ на $18 \%$ от 5.2 до $4.2 \mathrm{eV} /$ atom.

DOI: 10.21883/PJTF.2018.03.45573.16980

В последнее время благодаря новому поколению наноструктурированных бейнитных сталей было достигнуто уникальное сочетание прочности $\left(\sigma_{T} \approx 2.1 \mathrm{GPa}\right)$, вязкости $\left(K_{I C} \approx 34 \mathrm{MPa} \cdot \mathrm{m}^{1 / 2}\right)$ и пластичности $(\delta \approx 7 \%)$. Для получения подобного уровня свойств необходимо подавить выделение карбидов при образовании бейнита. Для достижения этой цели Бхадешия предложил [1] вводить в сталь кремний (до 2 wt.\%), a в работе [2] был показан практический эффект такого введения. В результате снова возник интерес к легированию сталей кремнием.

В работе [3] представлен обзор экспериментальных данных, согласно которому с ростом концентрации кремния до 6 at.\% происходит слабое уменьшение параметра ОЦК-решетки на $0.22 \%$. Обычно стали содержат менее 5 at.\% (или 2 wt.\%) Si. В работе [4] было рассчитано взаимодействие атомов углерода и кремния, находящихся на различных расстояниях в кристаллической решетке железа. Логично было бы, 
опираясь на эти данные, рассмотреть влияние примесей кремния на энергетику упорядочения атомов углерода в нетермоупругом [5] мартенсите. Поэтому было решено провести компьютерное моделирование данной системы методом молекулярной динамики (MD) с использованием межчастичных потенциалов ЕАМ-типа (EAM - embedded atom method), обеспечивающих достаточно точное воспроизведение свойств рассматриваемой системы.

Потенциальная энергия системы описывалась в рамках модели потенциала погруженного атома (EAM) [6], которая при подгонке к данным $a b$ initio моделирования обеспечивает достаточную реалистичность расчета. В рамках модели ЕАМ потенциальная энергия $i$-го атома сорта $\alpha$ имеет вид

$$
U_{\alpha . i}=\frac{1}{2} \sum_{j \neq i} \phi_{\beta \alpha}\left(r_{i j}\right)+F_{\alpha}\left(\rho_{i}\right),
$$

где первое слагаемое представляет собой парное взаимодействие ионов, а второе - взаимодействие иона с остальной системой посредством энергии погружения. Полная плотность, создаваемая на расстоянии $r$ от $i$-го иона, равна сумме

$$
\rho_{i}=\sum_{j \neq 1} \psi_{\beta \alpha}\left(r_{i j}\right)
$$

где $\psi(r)$ - электронная плотность, создаваемая атомом $j$ типа $\beta$ в точке, где находится атом $i$ сорта $\alpha$.

Для построения потенциалов межчастичного взаимодействия в тройных сплавах системы $\mathrm{Fe}-\mathrm{Si}-\mathrm{C}$ с ОЦК-решеткой нами предлагается следующая модель. В качестве основы принимается потенциал ЕАМ-типа для бинарной системы $\mathrm{Fe}-\mathrm{C}$ [7], который, как было показано ранее [8], позволяет адекватно описать взаимодействие внедренных атомов углерода в ОЦК-железе, приводящее к возникновению тетрагональной решетки мартенсита. Согласно ab initio расчетам, проведенным в [9], внедренные атомы $\mathrm{Si}$ практически не искажают кристаллическую решетку $\alpha$-железа и не вызывают изменений в ближнем порядке. Кроме того, наблюдается сильное отталкивание между атомами кремния и углерода в первых двух координационных сферах. Эти результаты позволяют нам считать, что потенциалы взаимодействия пар $\mathrm{Si}-\mathrm{Fe}$ и $\mathrm{Si}-\mathrm{Si}$ не слишком сильно отличаются от потенциала взаимодействия атомов железа $\mathrm{Fe}-\mathrm{Fe}$. Поэтому мы предположили выполнение для этих потенциалов,

Письма в ЖТФ, 2018, том 44, вып. 3 
Энергия связи $\mathrm{Si}-\mathrm{C}($ в $\mathrm{eV}$ ) в решетке $\alpha$-железа в различных координационных сферах (приведены результаты для построенного потенциала и доступные данные $a b$ initio расчетов, которые использовались при оптимизации)

\begin{tabular}{l|l|c|c|c}
\hline \multirow{2}{*}{ Метод } & \multicolumn{4}{|c}{ Номер координационной сферы } \\
\cline { 2 - 5 } & 1 & 2 & 3 & 4 \\
\hline DFT [4] & 0.478 & 0.75 & -0.021 & -0.057 \\
EAM, MD & 0.54 & 0.8 & 0.02 & 0
\end{tabular}

Примечание. DFT - теория функционала плотности.

определяемых общей формулой (1), равенства соответствующих функций: $F_{\mathrm{Si}}=F_{\mathrm{Fe}}, \phi_{\mathrm{FeSi}}=\phi_{\mathrm{FeFe}}, \psi_{\mathrm{FeSi}}=\psi_{\mathrm{SiFe}}=\psi_{\mathrm{FeFe}}$. Поскольку пары атомов $\mathrm{Si}-\mathrm{C}$ не могут сближаться на расстояние перекрывания электронных орбиталей, для потенциала их взаимодействия (1) сохраняли лишь парную часть, обращая в нуль соответствующие функции электронных плотностей: $\psi_{\mathrm{CSi}}=\psi_{\mathrm{SiC}}=0$.

Взаимодействие $\mathrm{Si}-\mathrm{C}$ подгонялось под данные $a b$ initio моделирования [4], причем рассматривалось приближение парного потенциала, поэтому соответствующие функции электронных плотностей были обращены в нуль: $\psi_{\mathrm{CSe}}=\psi_{\mathrm{SiC}}=0$. А парное взаимодействие $\phi_{\mathrm{SiC}}(r)$, представляющее собой серию сплайнов с непрерывными первой и второй производными, было оптимизировано под значения энергии связи $E_{\mathrm{SiC}}^{b}(\rho)$ кремния с углеродом, которая рассчитывается как

$$
\begin{aligned}
E_{\mathrm{SiC}}^{b}(\rho)= & {\left[E_{\rho}((N-1) \mathrm{Fe}+\mathrm{Si}+\mathrm{C})-E(N \mathrm{Fe}+\mathrm{Si})\right] } \\
& -[E((N-1) \mathrm{Fe}+\mathrm{Si}-E(N \mathrm{Fe}))],
\end{aligned}
$$

где $E(N \mathrm{Fe})$ - энергия чистой кристаллической решетки, содержащей $N$ атомов железа; $E((N-1) \mathrm{Fe}+\mathrm{Si})$ и $E(N \mathrm{Fe}+\mathrm{C})$ представляют собой энергии систем, содержащих атом замещения кремния и внедренный в октапору решетки атом углерода соответственно. Через $E_{\rho}((N-1) \mathrm{Fe}+\mathrm{Si}+\mathrm{C})$ обозначена энергия суперъячейки с атомами кремния и углерода, находящимися на расстоянии $\rho$. При таком способе расчета положительные значения $E_{\mathrm{SiC}}^{b}(\rho)$ соответствуют отталкиванию, а отрицательные - притяжению между примесями кремния и углерода. Из таблицы видно, что потенциал удалось достаточно точно оптимизировать под доступные нам результаты $a b$ initio моделирования. На рис. 1

Письма в ЖТФ, 2018, том 44, вып. 3 


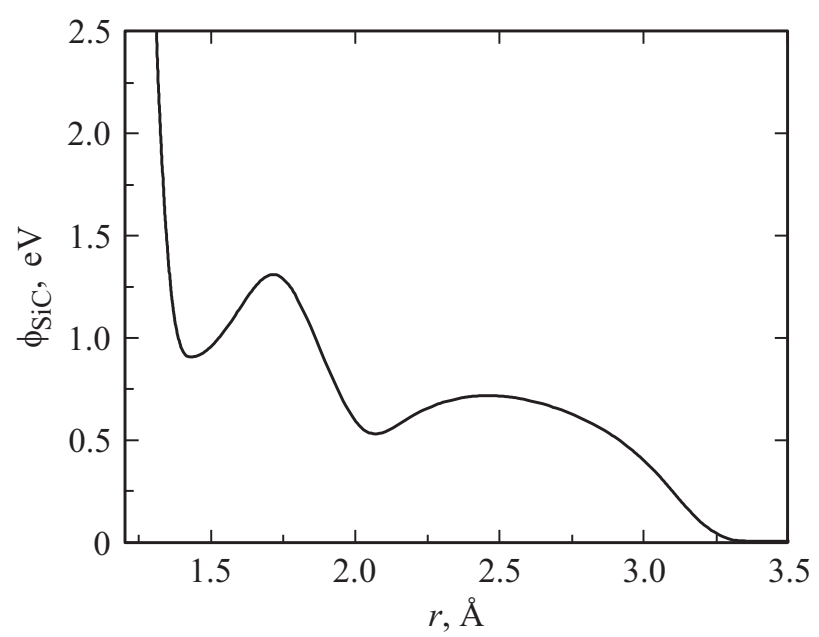

Рис. 1. Вид функции парного взаимодействия $\mathrm{Si}-\mathrm{C}$.

представлен вид оптимизированной функции парного взаимодействия $\phi_{\mathrm{SiC}}(r)$, положение локальных минимумов соответствует двум первым конфигурациям.

Свойства мартенсита $\mathrm{Fe}-\mathrm{Si}-\mathrm{C}$, например параметры решетки и ее тетрагональное искажение, согласно теории Зинера-Хачатуряна [10], зависят от распределения атомов углерода по октапорам различных подрешеток, и эта зависимость определяется величиной параметра деформационного взаимодействия $\lambda_{0}$. Однако влияние примесей кремния на величину этого параметра до сих пор остается неизученным.

Нами ранее было показано [8], что величину параметра $\lambda_{0}$ в рамках атомистического моделирования можно определить с помощью вытекающего из теории Зинера и Хачатуряна [10] выражения для свободной энергии мартенсита

$$
\begin{aligned}
F\left(c_{\mathrm{C}}, \eta\right)= & F\left(c_{\mathrm{C}}, 0\right)-\frac{1}{3} N \lambda_{0} c_{\mathrm{C}}^{2} \eta^{2}+\frac{2}{3} N c_{\mathrm{C}} k T \\
& \times[(1-\eta) \ln (1-\eta)+(1+2 \eta) \ln (1+2 \eta)],
\end{aligned}
$$

где $N$ - количество атомов в кристаллической решетке, а $c_{\mathrm{C}}-$ доля атомов углерода из этого числа. Параметр порядка $\eta$ показывает

Письма в ЖТФ, 2018, том 44, вып. 3 


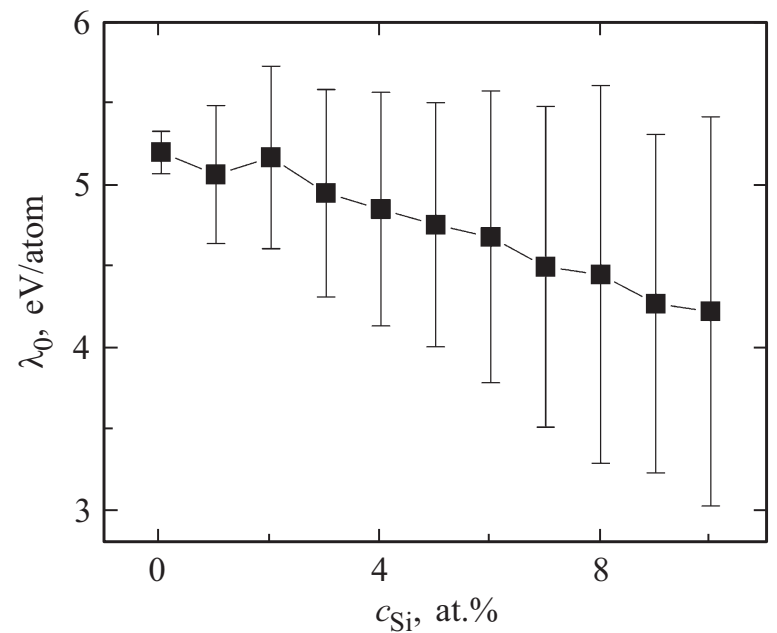

Рис. 2. Зависимость параметра деформационного упорядочения от концентрации кремния.

избыток атомов углерода в $z$-октаэдрических порах по сравнению с тем количеством, которое должно быть при равномерном заполнении атомами углерода пор типа $x, y \quad$ и $z$. Параметр порядка может изменяться от нуля (неупорядоченное состояние) до единицы (полное упорядочение).

Если мартенсит полностью упорядочен, то

$$
F\left(c_{\mathrm{C}}, 1\right)=F\left(c_{C}, 0\right)+E_{z}+k_{B} T N c_{\mathrm{C}} \ln 3,
$$

где $E_{z}=-\frac{N}{3} \lambda_{0} c_{\mathrm{C}}^{2}$ представляет собой энергию деформационного взаимодействия атомов углерода при полном упорядочении, т. е. все атомы углерода находятся в $z$-подрешетке, $\eta=1$. В свою очередь

$$
F(c, 0)=E_{x y z}-k_{B} T N c_{\mathrm{C}} \ln 3,
$$

где $E_{x y z}$ - энергия взаимодействия атомов углерода в неупорядоченном феррите. На основе указанных выражений получим формулу

$$
\lambda_{0}=3 \frac{E_{x y z}-E_{z}}{N c_{\mathrm{C}}^{2}} .
$$

Письма в ЖТФ, 2018, том 44, вып. 3 


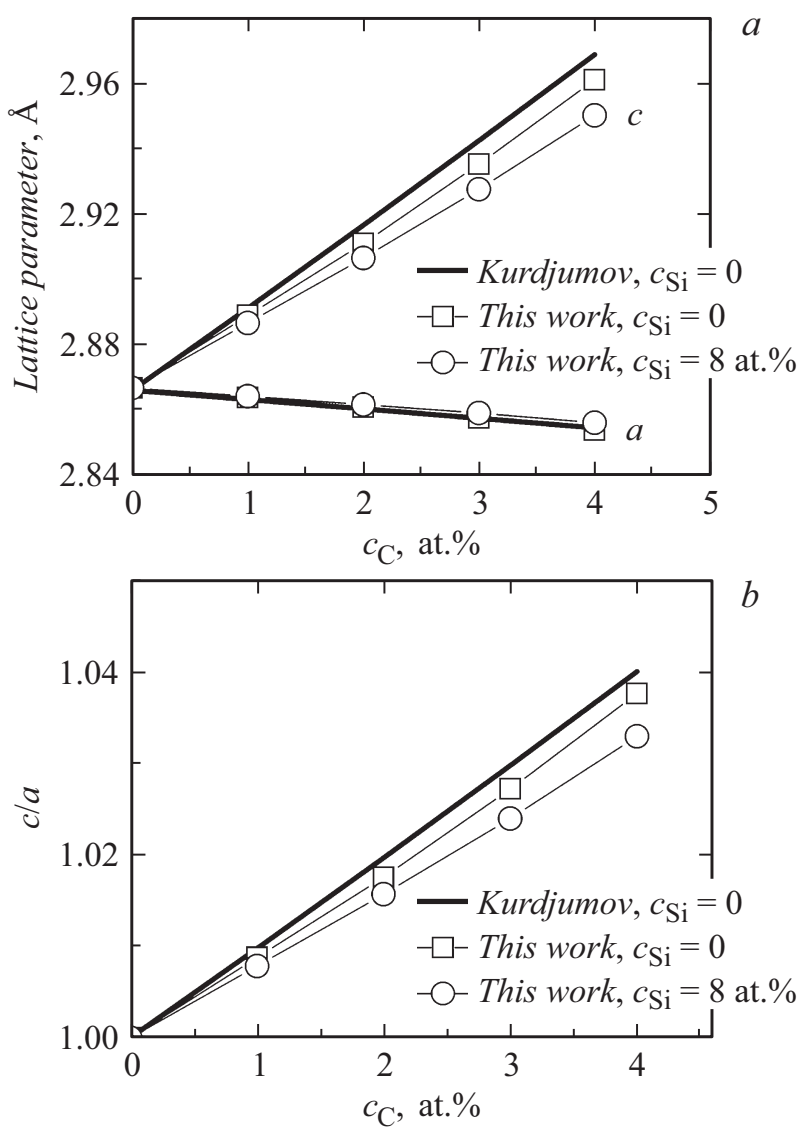

Рис. 3. Зависимости параметров решетки $(a)$ и степени тетрагональности $(b)$ от концентрации углерода. Представлены экспериментальные данные Курдюмова [11] и данные, полученные при MD-моделировании с помощью ЕАМ-потенциалов для содержания кремния 0 и 8 at.\%.

Расчет энергий $E_{x y z}$ и $E_{z}$ производился методом минимизации энергии суперъячейки, представляющей собой ОЦК-решетку железа размером $30 \times 30 \times 30$ элементарных ячеек $(54000$ атомов). Атомы кремния и углерода случайно распределяли по узлам кристаллической

Письма в ЖТФ, 2018, том 44, вып. 3 
решетки и октапорам соответственно. Энергии $E_{x y z}$ и $E_{z}$ усреднялись по 100 конфигурациям, содержание углерода было выбрано равным 4 at.\%. Заметим, что, согласно теории Зинера-Хачатуряна, параметр деформационного взаимодействия не зависит от содержания углерода. При увеличении концентрации кремния происходит уменьшение параметра $\lambda_{0}$ (рис. 2) вплоть до $4.2 \mathrm{eV} /$ atom (-18\%) при 10 at.\% Si, что, вероятно, обусловлено сильным отталкиванием атомов кремния и углерода, находящихся в близких координационных сферах.

На рис. 3 приведены концентрационные зависимости параметров $c$ и $а$ решетки мартенсита для растворов $\mathrm{Fe}-\mathrm{C}$ и $\mathrm{Fe}-\mathrm{C}-\mathrm{Si}$ c $10 \mathrm{at} . \% \mathrm{Si}$, а также экспериментальные данные Курдюмова [11]. Представленные зависимости получены при расчетах энергии $E_{z}$. Заметно, что введение кремния уменьшает параметры решетки и соответственно степень тетрагональности мартенсита $\mathrm{Fe}-\mathrm{Si}-\mathrm{C}$.

Таким образом, в работе предложены межчастичные потенциалы типа погруженного атома для мартенсита системы $\mathrm{Fe}-\mathrm{Si}-\mathrm{C}$, которые корректно воспроизводят данные $a b$ initio моделирования энергии взаимодействия между атомами кремния и углерода, находящимися на различных расстояниях в решетке $\alpha$-железа. Моделирование степени тетрагональности мартенсита кремнистых сталей, проведенное методом молекулярной динамики, позволило установить, что добавки кремния заметно уменьшают параметр $c$ и слабо увеличивают параметр $a$. Отношение $c / a$ понижается относительно классической кривой Курдюмова при всех содержаниях углерода. С использованием теории Зинера-Хачатуряна проведен расчет зависимости параметра деформационного взаимодействия атомов углерода в тройном растворе $\mathrm{Fe}-\mathrm{C}-\mathrm{Si}$ от содержания кремния. Увеличение концентрации кремния в пределах до 10 at.\% приводит к монотонному понижению параметра от 5.2 до $4.2 \mathrm{eV} /$ atom.

Исследование выполнено за счет гранта Российского научного фонда (проект № 16-19-10252).

\section{Список литературы}

[1] Bhadeshia H.K.D.H., Edmonds D.V. // Met. Mater. Trans. A. 1979. V. 10. N 7. P. 895-907.

[2] Garcia-Mateo C., Caballero F.G. // ISIJ International. 2005. V. 45. N 11. P. $1736-1740$.

Письма в ЖТФ, 2018, том 44, вып. 3 
[3] Predel B. Fe-Si (Iron-Silicon). Berlin-Heidelberg: Springer, 1995. P. 1-6.

[4] Simonovic D., Ande C.K., Duff A.I. // Phys. Rev. B. 2010. V. 81, N 5. P. 054116.

[5] Белослудиева Е.С., Куранова Н.Н., Марченкова Е.Б., Попов А.Г., Пушин В.Г. // Письма в ЖТФ. 2016. Т. 42. В. 2. С. 37-43.

[6] Daw M.S. Foiles S.M., Baskes M.I. // Mater. Sci. Rep. 1993. V. 9. N 7-8. P. $251-310$.

[7] Lau T.T., Forst C.J.F. // Phys. Rev. Lett. 2007. V. 98. N 21. P. 215501.

[8] Чирков П.В., Мирзоев А.А., Мирзаев Д.А. // ФММ. 2016. Т. 117. В. 1. С. 1-8.

[9] Vincent E., Becquart C.S., Domain C. // J. Nucl. Mater. 2006. V. 351. N 1-3. P. 88-99.

[10] Khachaturyan A.G. Theory of structural transformations in solids. Dover Publications, 2008. 576 p.

[11] Курдюмов Г.В., Утевский Л.М., Энтин Р.И. Превращения в железе и стали. М.: Наука, 1977. 236 с. 